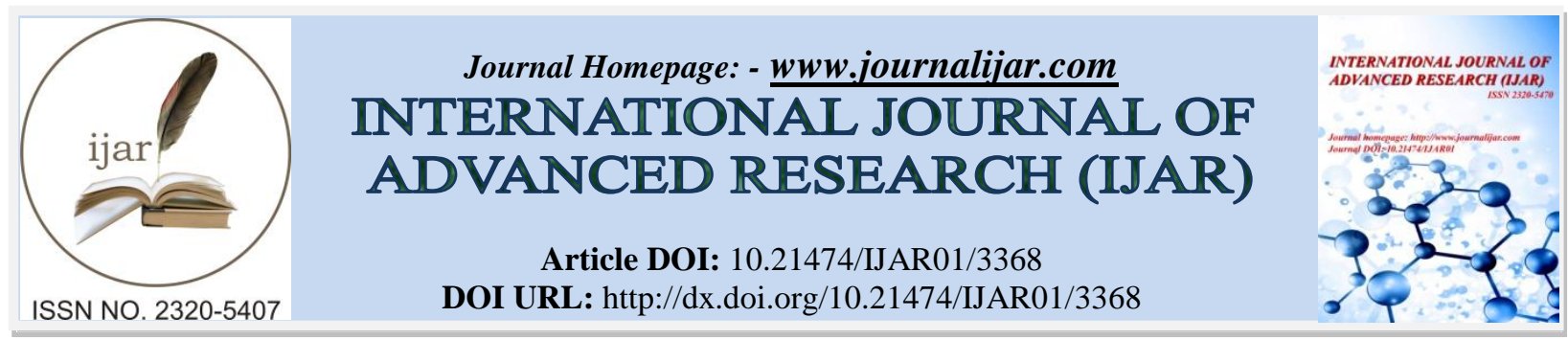

RESEARCH ARTICLE

\title{
OPTICAL AND THERMAL STUDIES ON NANO-MOLECULAR SELF-ASSEMBLED INDUCED SMECTIC PHASES OF LIQUID CRYSTALLINE MATERIALS.
}

\author{
T. N. Govindaiah ${ }^{1 *}$ and B. N. Ramakrishna ${ }^{2}$.
}

1. Research Centre, Post-Graduate Department of Physics, Government College (Autonomous), Mandya-571401, India.

2. Department of Physics, Government College for Women, Mandya-571401, India

\section{Manuscript Info}

Manuscript History

Received: 21 December 2016

Final Accepted: 16 January 2017

Published: February 2017

Key words:-

Molecular orientation, Optical textures, Helical pitch, Temperature dependence, Electrical conductivity.

\section{Abstract}

In the present work, our investigation is to study the optical and thermal properties of the binary mixture of nematic and cholesteric compounds namely, 2-cyanonaphthalen-6-yl 4-(3, 7-dimethyloctyloxy)-benzoate $(\mathrm{CNDOB})$ and cholesteryl nanonate $(\mathrm{CN})$, which exhibits different liquid crystalline phases of Cho-SmA-SmC-SmC*-SmE phases and it is obtained sequentially when the specimen is cooled from isotropic phase. These phases have been characterized by using X-ray and optical texture studies. The temperature variation of electrical conductivity and helical pitch of the cholesteric phase has been discussed.

Copy Right, IJAR, 2017,. All rights reserved.

\section{Introduction:-}

Liquid Crystals are a state of matter that is intermediate between the crystalline solid and amorphous liquid. Liquid crystals are flow like ordinary liquids and also exhibit anisotropy in their optical, electrical, magnetic properties like crystals. They are also called as mesophases because of their intermediate nature. Liquid crystalline materials are broadly classified as thermotropics and lyotropics. In thermotropics, the phase transition is mainly controlled by temperature: whereas in lyotropics, apart from temperature: phase transitions are controlled by the concentrations of molecules. On the basis of molecular arrangement, thermotropics are further classified into three main types namely, nematic, smectic and cholesteric. Nematics are characterized by orientational order while smectics have an additional positional order. Cholesterics are similar to nematics but, chiral in structure.

The cholesteric phase is regarded as twisted nematic wherein the molecules are orientationally ordered, but at the same time they are rotationally disordered withrespect to long axis [1-3]. It is well known that, if a small percentage of cholesteric liquid crystals added to nematic, it results in the helical direction and pitch of the phase increases. When the pitch is comparable to wavelength of light the phase become iridescent because of the selective reflections of light. The recent studies on the mixture of cholesteric and nematic liquid crystals reveals that the mixture exhibits frustrated blue phase, twisted grain boundary phase, tilted phase, cholesteric phase and quasi crystalline phase[4-6]. Because of the tremendous potentialities of the liquid crystals in the field of display device technology, we have proposed the studies on the optical and thermal properties of mixture of cholesteric and nematic liquid crystals. Optical, thermal and X-ray studies have been carried out to understand the intermolecular interactions and the name of the induced smectic phases exhibited by the mixture.

Corresponding Author:- T. N. Govindaiah.

Address:- Research Centre, Post-Graduate Department of Physics, Government College (Autonomous), Mandya-571401, India. 


\section{Experimental section:-}

In the present investigation, we have been considered the binary mixture of liquid crystalline materials, namely: cholesteryl nanonate $(\mathrm{CN})$ and 2-cyanonaphthalen-6-yl 4-(3,7- dimethyloctyloxy)-benzoate (CNDOB). The chemicals are purified twice with benzene. Mixtures of twenty different concentrations of CN in CNDOB were prepared and were mixed thoroughly. These mixtures of various concentrations of $\mathrm{CN}$ in CNDOB were kept in desiccators for a long time. The samples were subjected to several cycles of heating, stirring and centrifuging to ensure homogeneity. The phase transition temperatures of these concentrations were measured with the help of Leitz-polarizing microscope in conjunction with a hot stage. The samples were sandwiched between the slide and cover slip and were sealed for microscopic observations. The X-ray broadening peaks were obtained at different temperatures using JEOL diffractometer. The density and refractive indices in the optical region are determined at different temperatures by employing the techniques described by the earlier investigators [7, 8]. Electricalconductivity measurements of the mixture at different temperatures were carried out using digital LCR meter and a proportional temperature control unit.

\section{Results and Discussions:- \\ Phase Diagram:-}

The partial phase diagram of given molecules is a very important method to determine the stability of liquid crystalline phase at different temperatures for different concentrations. The partial phase diagram in the present case is as shown in Figure 1. This clearly illustrates that, the mixtures with concentrations ranging from 5\% to $85 \%$ of $\mathrm{CN}$ in CNDOB exhibit Cholesteric $\mathrm{SmA}, \mathrm{SmC}, \mathrm{SmC}^{*}$ and $\mathrm{SmE}$ phases sequentially when the specimen is cooled from its isotropic melt. But the mixtures with concentrations ranging from 10\% to 65\% of CN in CNDOB show only SmC* phase in addition to the above phases respectively at different temperatures. [9].

\section{Optical Texture Studies:-}

For the purpose of optical texture studies, the sample was sandwiched between the slide and cover glass and then the optical textures were observed using Leitz-polarizing microscope in conjunction with hot stage. The concentrations ranging from $10 \%$ to $65 \%$ of the given mixture are slowly cooled from its isotropic melt. The genesis of nucleation starts in the form of small bubbles and slowly grow radially, which form a spherulitic texture of cholesteric phase with large values of pitch and texture is as shown in Figure 2(a) [10-12] at temperature $94{ }^{\circ} \mathrm{C}$. On further cooling the specimen, the cholesteric phase slowly changes over to focal conic fan shaped texture, which is the characteristics of SmA phase and is as shown in Figure 2(b) at temperature $80{ }^{\circ} \mathrm{C}$. On further cooling the specimen, SmA phase changes over to schlieren texture of SmC phase, which is as shown in Figure 2 (c) at temperature $65{ }^{\circ} \mathrm{C}$. The SmC phase is not stable and then changes over to $\mathrm{SmC}^{*}$ phase, which exhibits radial fringes on the fans of focal conic textures, which is characteristic of chiral SmC* phase. Further cooling the specimen, the chiral SmC* phase changes over to the crystalline SmE phase, which remains up to room temperature and then it, becomes crystalline phase.

\section{Conductivity Measurements:-}

Electrical-conductivity measurements help in getting better idea on the phase behavior with temperature. An abrupt increase or decrease of electrical-conductivity with temperature relates to the phase behavior of the lyotropic and thermotropic systems [13].The temperature variation of electrical-conductivity for the sample of $40 \% \mathrm{CN}$ in CNDOB is shown in Figure 3. The changes were observed in electrical-conductivity, the values correspond to liquid crystalline phase transition of thermotropic and lyotropic systems, respectively, at different temperatures and they were also identified by optical texture studies. It was observed that, the electrical-conductivity shows changes as we move from $96.5^{\circ} \mathrm{C} \rightarrow 90.5^{\circ} \mathrm{C}, 90.5^{\circ} \mathrm{C} \rightarrow 70^{\circ} \mathrm{C}, 70^{\circ} \mathrm{C} \rightarrow 60^{\circ} \mathrm{C}$, and $60^{\circ} \mathrm{C} \rightarrow 51^{\circ} \mathrm{C}, 51^{\circ} \mathrm{C} \rightarrow 48^{\circ} \mathrm{C}$ which correspond to phase transitions $\mathrm{Ch} \rightarrow \mathrm{SmA}, \mathrm{SmA} \rightarrow \mathrm{SmC}, \mathrm{SmC} \rightarrow \mathrm{SmC}^{*}, \mathrm{SmC}^{*} \rightarrow \mathrm{SmE}$ and $\mathrm{SmE} \rightarrow$ Cryst phases respectively. This type of behavior is generally observed in hexagonal, cubic and lamellar phases of lyotropic and thermotropic systems [14, 15]. These abrupt changes cannot be throughout if only due to change in the orientation of molecules. They can be attributed to changes in the dimension of disk along with changes in orientation.

\section{Helical Pitch Measurements In Smectic And Cholesteryl Layers:-}

The helical pitch measurements were performed on the cholesteric phase following the well-known Grandjean-Cano wedge method $[16,17]$. The given mixture was taken in a wedge-shaped cell treated for homogeneous alignment. The two glass plates formed a small angle at the wedge. The mixture was cooled slowly $\left(0.2^{\circ} \mathrm{C} \mathrm{min}{ }^{-1}\right)$ from isotropic cholesteric to smectic phase, which induces an array of equidistant Grandjean-Cano lines. The pitch of cholesteric phase was determined by measuring the distance between the Grandjean-Cano lines as a function of temperature. As the temperature was lowered the mesophase changes from cholesteric to smectic phase and the 
spacing between the lines are increased, indicating that the pitch in the cholesteric phase is also increasing. The temperature variation of pitch for the mixture of $40 \% \mathrm{CN}$ in $\mathrm{CNDOB}$ is shown in Figure 4. From this figure, it is evident that, the variation of pitch from cholesteric to smectic phase is smooth and continuous. But gradually, the value of pitch increases from 0.17 to $0.19 \mathrm{~mm}$ upon cooling the sample from cholesteric to smectic phase. The value of the pitch increases steeply and reaches a maximum of $0.34 \mathrm{~mm}$ at the cholesteric to smectic phase transition. In this study, we have noticed that, the sequence is Iso $\rightarrow \mathrm{Cho} \rightarrow \mathrm{SmA} \rightarrow \mathrm{SmC} \rightarrow \mathrm{SmC} * \rightarrow \mathrm{SmE}$ on cooling. Most of the data about the helical pitch are available in literature [18]. The pitch is continuous at the cho $\rightarrow$ smectic transition in spite of a rather energetic transition. It increases on cooling to smectic phase and diverges on approaching the SmA, $\mathrm{SmC}, \mathrm{SmC} *$ and SmE phases. This divergence is related to the second order nature of the transition. It exhibits a steep decrease, close to cholesteric phase which is usually the characteristics of second-order $\mathrm{SmA}, \mathrm{SmC}, \mathrm{SmC} *$ and SmE phase transitions.

\section{X-Ray Studies:-}

To understand the change in layer spacings in SmA and SmC phases with respect to temperature, $\mathrm{X}$-ray diffractometer traces were taken. The traces obtained for the mixture of $40 \% \mathrm{CN}$ in $\mathrm{CNDOB}$ at different temperatures correspond to $\mathrm{SmA}$ and $\mathrm{SmC}$ phases. It is observed that as the temperature increases the layer spacing also increases in SmC phase. But in SmA phase the layer spacing's are almost constant. These variations are as shown in Figure $5[15,16]$.

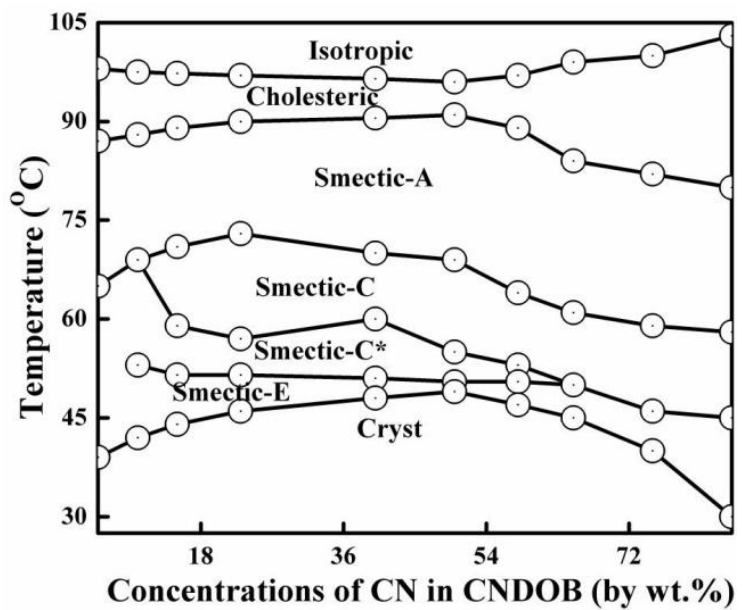

Figure 1:- Partial phase diagram for the mixture of $\mathrm{CN}$ in $\mathrm{CNDOB}$.

Figure 2:- Microphotographs obtained in between the crossed polars.

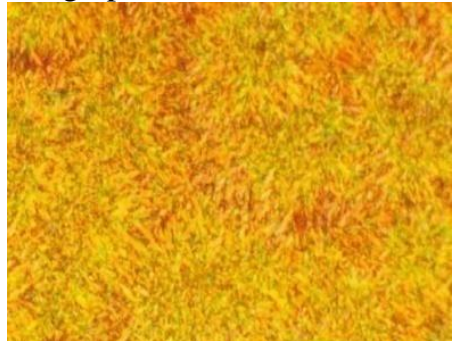

a) Spherulitic texture of cholesteric phase at temperature $94{ }^{0} \mathrm{C}$. 


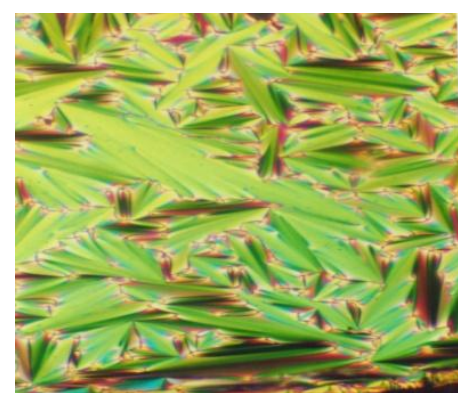

b) Focal conic fan shaped texture of Smectic-A phase at temperature $80{ }^{\circ} \mathrm{C}$.

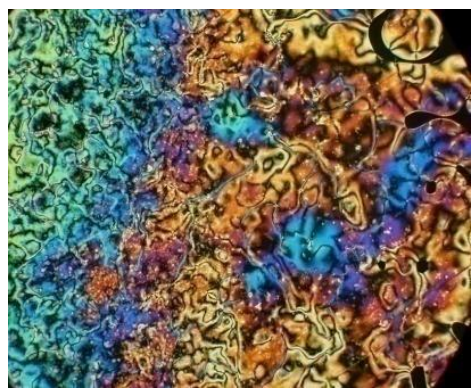

c) Schlieren texure of Smectic-C phase at temperature $65^{\circ} \mathrm{C}$.

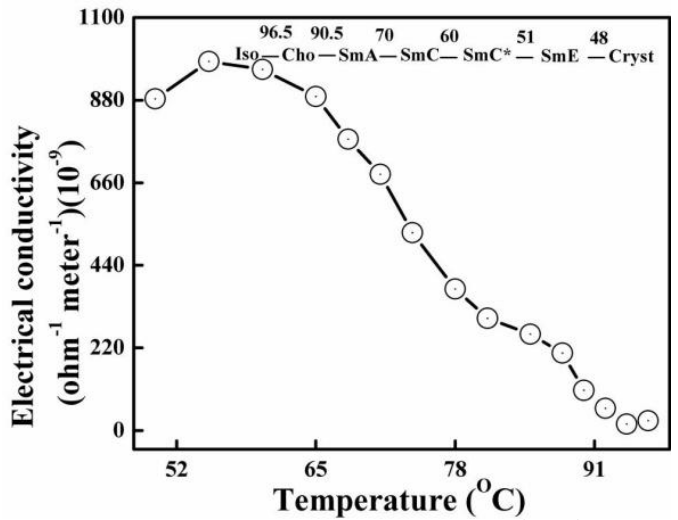

Figure 3:- Temperature variation of electrical-conductivity $\sigma\left(\times 10^{-9} \Omega^{-1} \mathrm{~m}^{-1}\right)$ for the sample of $40 \% \mathrm{CN}$ in CNDOB.

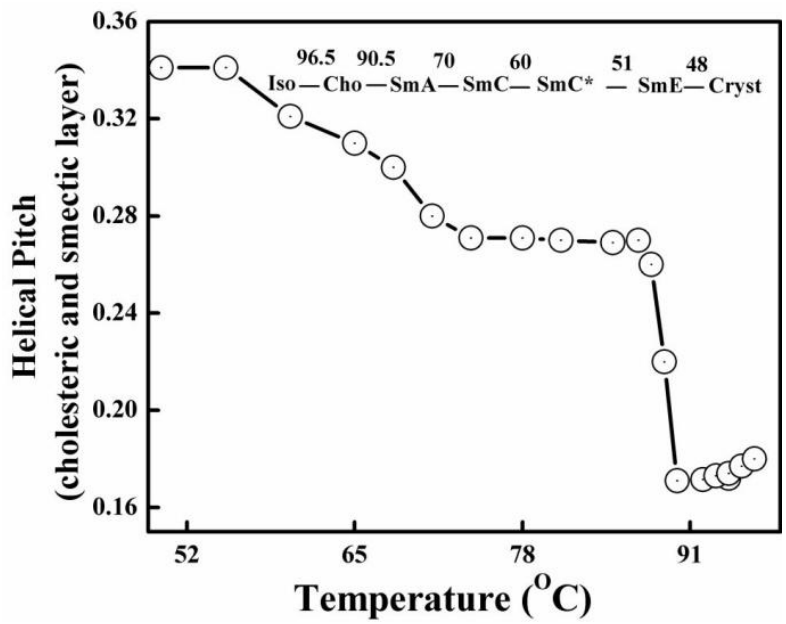

Figure 4:- The temperature variations of pitch for the mixture of $40 \% \mathrm{CN}$ in CNDOB. 


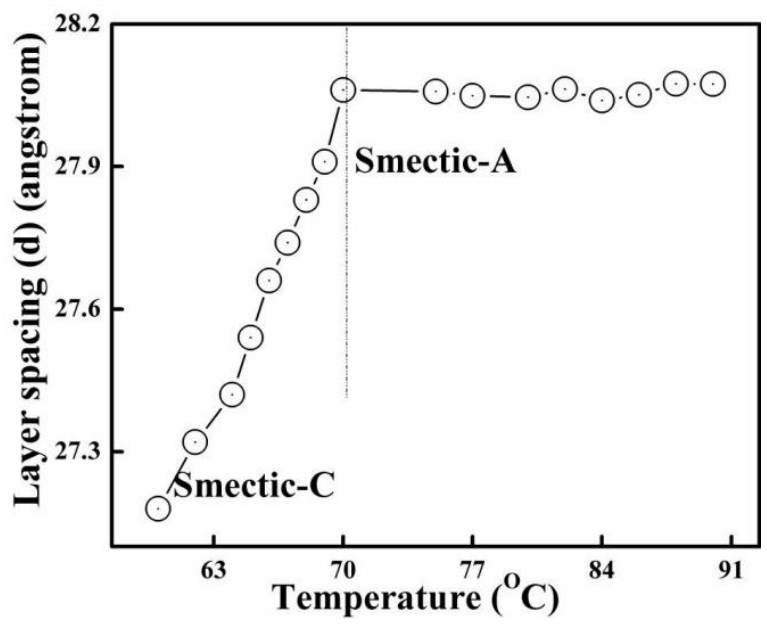

Figure 5:-Variation of layer spacing with temperature for the sample of $40 \% \mathrm{CN}$ in CNDOB.

\section{Conclusions:-}

In light of the above results, we have drawn the following conclusions. The binary system of given mixture exhibits an unusual sequence of phases showing the formation of cholesteric, SmA, SmC, SmC* and SmE phases in different concentrations of $\mathrm{CN}$ in CNDOB. The phase behavior is discussed with the help of phase diagram. The X-ray results also lend support to the above observations. Changes in the values of electrical conductivity with temperature suggest that the size of aggregated molecules goes on increasing and the electrical conductivity is also increasing, while the mixture is cooled from the isotropic phase. The pitch of cholesteric phase is continuously increasing at the transition from cholesteric to smectic phase transition. But, it is very interesting to see that it increases on cooling to smectic phase, which evidently diverges on approaching the SmA, SmC, SmC*and SmE phases respectively at different temperatures.

\section{References:-}

1. Friedel, G. (1922). The Mesomorphic States of Matter Annales de Physique 18, $273-474$.

2. De Gennes, P. G. and Prost, J. 1993. The Physics of Liquid Crystals, Chapter-1 (OxfordUniversity Press).

3. Govindaiah, T. N. and Sreepad, H. R. 2015. Phase transition and thermal characterization of induced smectic phases in a ternary mixture. Molecular Liquids, 202, 75-78.

4. Nagappa, Revanasiddaiah,D. Krishnamurti, D. Optical Behaviour of Mixtures of Nematic and Cholesteric Compounds. Mol. Cryst. Liq. Cryst. 1983, 101(1-2); 103-127.

5. Theim J, Vill V, Fischer F. Synthesis, properties and conformations of liquid crystalline 1,4-dioxanes and comparison with the corresponding 1,4-dithiane, cyclohexane, and benzene derivatives. Mol Cryst Liq Cryst. 1989;170:43-51.

6. Govindaiah, T. N. 2016. Temperature-dependent Anisotropic Nano-Molecular Orientations of Liquid Crystalline Materials. Mol. Cyst.Liq. Cryst. 626, 151-159.

7. Marthandappa M, Nagappa, Somashekar R, Lokanatha Rai KM. Mesomorphic behaviour of binary mixtures of two non-mesogenic compounds. Physica Status Solidi (A). 1992;129:389-398.

8. Govindaiah, T. N., Sreepad, H. R., Sathyanarayana, P. M., Mahadeva, J. and Nagappa 2012. Micellar nematic phase in a binary mixture of two nonmesogenic compounds. Mol. Cryst. Liq. Cryst. 552, 24-32.

9. Govindaiah, T. N., Sreepad, H.R. and Nagappa. 2013. Optical and thermal characterization of binary mixtures of liquid crystals. Mol. Cyst.Liq. Cryst. 574, 9-18.

10. Demus, D. and Richter, C. (1978). Textures of Liquid Crystals. Verlag Chemie,Weinheim, New York.

11. Govindaiah, T. N. 2016. Nano segregation and thermal stability of TGB and re-entrant smectic-A phases in a ternary mixture of liquid crystalline materials. Mol. Cryst. Liq. Cryst. 625, 93-98.

12. Nagappa, Nataraju S K, Krishnamurti D. Optical behavior of natural oils and fats of plant origin.Mol Crystals Liquid Crystals. 1986, 133, 31-54.

13. Marthandappa, M., Nagappa and Lokhanatha Rai, K. M. (1991). Thermotropic liquid- crystalline phases in binary mixtures of nonmesogenic compounds J. Phys. Chem 95(16), 6369-6372. 
14. Govindaiah, T. N., Sreepad, H. R. and Nagappa. (2014). Thermal characterization and mesomorphic properties of binary mixture of two non-mesogenic compounds. Mol. Cryst. Liq. Cryst.593,51-60.

15. Govindaiah, T. N., Sreepad, H. R., Nagappa, and Mahadeva, J. (2014).Optical characterization of lyotropic chromonic liquid crystalsMol. Cryst. Liq.Cryst.593, 43-50.

16. Sadashiva, B. K. (1999). Molecular structure and chiral liquid crystalline phases. Pramana J. Phys. 53, 213-222.

17. Govindaiah, T. N., Sreepad, H. R. and Nagappa. (2013).Thermal characterization of induced mesomorphism in binary mixture of cholesteryl and poly ethylene glycol. Mol. Cryst. Liq. Cryst. 575, 22-29.

18. Balasubramanian, D. (2005). Photodynamics of cataract: an update on endogenous chromophores and antioxidants. Photochem. Photobiol. 81, 498-501.

19. de Gennes, P. G. and Prost, J. (1975). The Physics of Liquid Crystals. Clarendon Press, Oxford.

20. Govindaiah, T. N. (2016). Phase transition and thermal stability of reentrant smectic phase in mixture of liquid crystalline materials. Mol. Cryst. Liq. Cryst. 625, 99-105. 